07,11

\title{
Механизм плавления при быстром нагреве
}

\author{
(С) Л.А. Булавин, Ю.Ф. Забашта, Л.Ю. Вэргун \\ Киевский национальный университет им. Тараса Шевченко, \\ Украина, Киев, \\ ๑ E-mail: verlen73@ukr.net \\ Поступила в Редакцию 28 февраля 2019 г. \\ В окончательной редакции 28 февраля 2019 г. \\ Принята к публикации 5 марта 2019 г.
}

\begin{abstract}
Рассматривается поведение идеального кристалла при быстром нагреве, когда время оседлой жизни частиц существенно превышает характерное время изменения температуры. В качестве расчетной модели выбран обладающий простой кубической решеткой кристалл, в котором взаимодействие между частицами описывается потенциалом Леннарда-Джонса. Принимается, что разупорядочение кристалла при нагреве является результатом образования вакансий. Показано, что при быстром нагреве вакансии образуются только в случае, если температура превышает некоторую критическую температуру $T_{C}$. Получено уравнение состояния, описывающее поведение кристалла с вакансиями при быстром нагреве. Установлено существование температуры, при которой кристаллическая решетка теряет механическую устойчивость. Эта температура отождествляется с температурой плавления, происходящего при быстром нагреве.
\end{abstract}

Ключевые слова: кристаллическая решетка, плавление, вакансионные кластеры, фазовый переход.

DOI: 10.21883/FTT.2019.07.47842.393

\section{1. Введение}

По определению (см. напр., [1] и др.), при заданном давлении $P=P_{M}$ температура плавления $T=T_{M}$ твердого тела (твердой фазы некоторого вещества) это температура, при которой находятся в равновесии твердая и жидкая фазы этого вещества, разделенные плоской поверхностью.

Различают два вида плавления.

Первый вид реализуется в случае, когда температура и давление одинаковы для всех участков твердого тела. В этих условиях плавление начинается с поверхности, как только температура достигает $T_{M}$, причем процесс плавления захватывает все твердое тело. Если же на поверхности твердого тела поддерживается температура, исключающая возможность плавления, а внутри находятся источник или источники тепла, то реализуется второй вид плавления, протекающего внутри твердого тела. Этот вид плавления изучается в классической теории фазовых превращений (см. напр., [1-3] и др.), где разработан следующий механизм плавления.

Флуктуационным путем в твердой фазе возникают зародыши жидкой фазы различного размера $R$. Появление зародыша приводит к изменению термодинамического потенциала на величину $\Delta \Phi$. Зависимость $\Delta \Phi(R)$ обладает максимумом при некотором критическом значении $R=R_{c}$. Соответственно такие критические зародыши способны произвольно расти. Увеличение количества жидкой фазы происходит за счет присоединения к растущему зародышу частиц окружающей его твердой фазы.

Вследствие этих процессов в системе устанавливается равновесие, при котором вся система оказывается состоящей из единой - жидкой фазы. Обозначим время установления указанного равновесия через $\tau_{N}$.
Обозначим через $v_{Q}$ скорость быстрого нагрева системы. Характерное время изменения температуры $\tau_{Q}$ определим равенством

$$
\tau_{Q}=\left(T_{M}-T_{B}\right) / v_{Q},
$$

где $T_{B}$ - температура, с которой начинается быстрый нагрев.

В теории [1-3] предполагается, что упомянутые процессы установления равновесия протекают при постоянной температуре. Это предположение можно записать в виде

$$
\dot{\tau}_{N} \ll \tau_{Q} .
$$

Как известно [1], поступательное движение частиц кристалла характеризуется временем оседлой жизни $\tau$, которое определяется формулой

$$
\tau=\tau_{0} \exp \left(\frac{\Delta u}{k_{B} T}\right) \ll \tau_{N},
$$

где $k_{B}$ - постоянная Больцмана, $\tau_{0}-$ период тепловых колебаний частицы, $\Delta u-$ активационный барьер, преодолеваемый частицей при каждом элементарном перемещении.

Обозначим через $\tau_{T}$ время установления равновесия между колебательными степенями свободы. Очевидным является неравенство

$$
\tau_{T} \ll \tau .
$$

В отличие от теории [1-3], где предполагается выполнение условия (2), в данной статье рассматривается плавление при температурном режиме, удовлетворяющем условию

$$
\tau_{T} \ll \tau_{Q} \ll \tau .
$$

Этим условием определяется используемый в данной статье термин „быстрый нагрев“. 
Актуальность изучения плавления при быстром нагреве состоит в том, что такая ситуация может возникнуть в ядерной энергетике, угрожая авариями.

\section{2. Особенности образования вакансий при быстром нагреве}

Выбирая тип статистического ансамбля для системы, находящейся в условиях быстрого нагрева, будем считать заданными энтропию и объем (см., напр. [4] и др.). Соответственно характеристической термодинамической функцией оказывается энергия $E$, которая в равновесии определяется выражением

$$
E_{e}=E_{e}^{\prime}(S, V) \text {. }
$$

Обозначим через $V_{0}$ объем системы в недеформированном состоянии, при котором в системе отсутствуют напряжения. Будем считать, что это состояние соответствует температуре $T=0$. Введем также обозначение для деформации

$$
\theta=\frac{V-V_{0}}{V_{0}} .
$$

Перепишем выражение (6) в виде

$$
E_{e}=E_{e}^{\prime \prime}(S, \theta) \text {. }
$$

Рост температуры приводит к возникновению беспорядка в кристалле. В данной статье для описания беспорядка используется вакансионная модель (см.,напр., [1] и др.). Соответственно в качестве дополнительного параметра, характеризующего состояние неполного равновесия, выберем количество вакансий $n$, записывая для энергии этого состояния выражение

$$
E_{n}=E_{n}^{\prime \prime}(S, \theta, n)
$$

Поскольку в силу условия (4) колебательная подсистема находится в состоянии равновесия, а $S$ - это энтропия состояния неполного равновеия, то последнюю можно связать только с поступательным движением: это - конфигурационная энтропия. Однако поступательное движение исключено неравенством (5), а это означает, что $S=0$ и выражение (9) приобретает вид

$$
E_{n}=E_{n}(\theta, n)
$$

Условие равновесия в этом случае

$$
\left.\frac{\partial E_{n}}{\partial n}\right|_{\theta=\mathrm{const}}=0 \text {. }
$$

Определенная из равенства (11) функция $n_{e}(\theta)$ должна удовлетворять условию

$$
E_{e}(\theta)=E_{n}\left(\theta, n_{e}(\theta)\right)
$$

При решении поставленной в данной статье задачи использована в видоизмененной форме расчетная модель, применявшаяся нами ранее в работах $[5,6]$ для других целей. Модель представляет собой простую кубическую решетку. Энергия взаимодействия между частицами $u$ имеет вид

$$
u=\varepsilon\left\{\frac{q}{n-q}(\lambda)^{-m}-\frac{m}{m-q}(\lambda)^{-q}\right\},
$$

где $\varepsilon-$ энергия разрыва межчастичной связи, $\lambda-$ степень деформации связи, определяемая формулой

$$
\lambda=a / a_{0}
$$

где $a_{0}$ - размер ячейки в недеформированном состоянии, когда одновременно $\theta=0$ и $n=0$, во всех остальных случаях (деформированное состояние) этот размер обозначается через $a$.

В соответствии с введенными обозначениями имеем

$$
V_{0}=N a_{0}^{3},
$$

где $N$ - число частиц.

Наличие $n$ вакансий в системе означает, что система содержит $(N+n)$ узлов. Соответственно для объема системы получаем

$$
V=(N+n) a^{3} .
$$

Сопоставляя равенства $(7,14,15)$, записываем

$$
\lambda=\left(\frac{1+c}{1+\theta}\right)^{1 / 3}
$$

Обозначим посредством $A$ и $B$ заполненную и пустую ячейки соответственно, через $N_{A A}, N_{A B}, N_{B B}$ - число контактов между соответствующими ячейками. Величина $N_{A A}$ - это число межчастичных связей, уцелевших после появления в системе $n$ вакансий, что позволяет для энергии системы $E_{n}$ записать формулу

$$
E_{n}=u N_{A A} .
$$

Общее количество контактов между ячейками определяется очевидной формулой

$$
3(N+n)=N_{A A}+N_{B B}+N_{A B} .
$$

Учтем то обстоятельство, что вакансии могут объединяться в кластеры. Принимая во внимание тип решетки, припишем кластерам форму куба. Обозначим через $x$ число кластеров, через $y^{3}-$ количество ячеек в кластере, в связи с чем для количества вакансий записываем выражение

$$
n=x y^{3} .
$$

Обозначая через $N_{B B}^{\prime}$ и $N_{A B}^{\prime}-$ количество соответствующих контактов между ячейками в кластере, получаем

$$
N_{B B}+N_{A B}=x\left(N_{B B}^{\prime}+N_{A B}^{\prime}\right) .
$$


Для кластера принятой нами кубической формы имеем

$$
\begin{gathered}
N_{A B}^{\prime}=6 y^{2}, \\
N_{B B}^{\prime}=3 y^{2}(y-1) .
\end{gathered}
$$

В случае, когда $y=1$, т.е. когда кластер превращается в единичную вакансию, имеем очевидное значение $N_{A B}=6, N_{B B}=0$.

Подставляя равенства (20-22) в формулу (19), получаем

$$
N_{A A}=3 N\left(1-\frac{c}{y}\right),
$$

где $c=n / N-$ концентрация вакансий.

После подстановки равенств (13) и (23) в формулу (18), приходим к выражению

$$
\begin{aligned}
E_{n}= & 3 N \varepsilon\left[\frac{q}{m-q}\left(\frac{1+c}{1+\theta}\right)^{m / 3}-\frac{m}{m-q}\left(\frac{1+c}{1+\theta}\right)^{q / 3}\right] \\
& \times\left(1-\frac{c}{y}\right) .
\end{aligned}
$$

Учитывая очевидное неравенство $c / y \ll 1$, приближенно запишем

$$
\left(1-\frac{c}{y}\right)=(1+c)^{-1 / y}
$$

после чего выражение (24) принимает вид

$$
\begin{aligned}
E_{n}= & 3 N \varepsilon\left[\frac{q}{m-q}(1+\theta)^{-m / 3}(1+c)^{m / 3-1 / y}\right. \\
& \left.-\frac{m}{m-q}(1+\theta)^{-q / 3}(1+c)^{q / 3-1 / y}\right] .
\end{aligned}
$$

Дифференцируя это выражение в соответствии с формулой (11), получаем уравнение

$$
\left(\frac{1+c}{1+\theta}\right)^{\frac{m-q}{3}}=\frac{m}{q} \frac{\left(\frac{q}{3}-\frac{1}{y}\right)}{\frac{m}{3}-\frac{1}{y}} .
$$

Его решение имеет вид

$$
c_{e}= \begin{cases}0, & \theta \leq \theta_{c} \\ \frac{\theta-\theta_{c}}{1+\theta_{c}}, & \theta>\theta_{c},\end{cases}
$$

где принято обозначение

$$
\theta_{c}=\left\{\frac{1-\frac{3}{m y}}{1-\frac{3}{q y}}\right\}^{\frac{3}{m-q}}-1 .
$$

В рамках рассмотренного статистического ансамбля температура входит в формулу посредством соотношения

$$
\theta(T)=\int_{0}^{T} \alpha\left(T^{\prime}\right) d T^{\prime},
$$

где $\alpha$ - коэффициент теплового расширения.
Для деформации $\theta_{c}$ имеем формулу

$$
\theta_{c}=\int_{0}^{T_{c}} \alpha\left(T^{\prime}\right) d T^{\prime} .
$$

Равенства (30-31) позволяют переписать выражение (28) в виде

$$
c_{e}= \begin{cases}0, & T \leq T_{c} \\ \frac{\theta-\theta_{c}}{1+\theta_{c}}, & T>T_{c} .\end{cases}
$$

В классической теории вакансий (см., напр., [1], [7] и др.), для $c_{e}$ получена другая формула

$$
c_{e}=\exp \left(-\frac{W}{k_{B} T}\right),
$$

где $W-$ энергия образования вакансии, $k_{B}-$ постоянная Больцмана. Между обеими формулами существует принципиальное различие: если из выражения (32) следует, что образование вакансий при быстром нагреве носит пороговый характер: они появляются в кристалле только тогда, когда температура превысит некоторую критическую температуру $T_{c}$, то согласно формуле (33), они обязаны возникать при любой температуре.

Причина этого различия коренится в различных температурных режимах, действию которых подвергается система в обоих случаях: при выводе формулы (33) предполагалось (снова, как и в теории фазовых переходов), что образование вакансий происходит при постоянной температуре, из чего следовало условие $S>0$. При быстром же нагреве $S=0$, что приводит к формуле (32).

Чтобы различать оба упомянутых случая, нам представляется логичным применять по отношению к ним соответственно термины „неклассические“ и „классические“ вакансии.

Произведем количественную оценку критической деформации $\theta_{c}$. Будем считать, что $u-$ потенциал Леннарда-Джонса, принимая соответственно $m=12$, $q=6$. Тогда для $y=1$ согласно формуле (29) имеем $\theta=0.22$. Однако уже для $y=2$ получаем $\theta_{c}=0.03$, а для кластеров, характеризуемых значениями $y=3$ и т. д., величина $\theta_{c}$ оказывается меньшей, чем 0.03 .

Согласно формулам (30-31) деформация $\theta$ в системе возникает вследствие теплового расширения. Согласно справочным данным для твердых тел с температурой плавления порядка $10^{3} \mathrm{~K}$ коэффициент теплового расширения имеет порядок $10^{-5} \mathrm{~K}^{-1}$. Соответственно деформация, создаваемая тепловым расширением при температуре $T<T_{M}$, по порядку величины равна нескольким сотым. Из этого следует, что образования единичных вакансий не происходит: при быстром нагреве образуются вакансионные кластеры - преимущественно малые, содержащие несколько вакансий (четыре - для кластеров кубической решетки). 


\section{3. Уравнение состояния и плавление при быстром нагреве}

Деформация создает в кристалле отрицательное давление

$$
P=-\left.\frac{\partial E_{n}}{\partial \theta}\right|_{c=\text { const }} .
$$

Деформация $\theta$ мала, что позволяет считать $E_{n}$ квадратичной функцией от $\theta$ и, соответственно, записать формулу

$$
P=-K \theta,
$$

где $K$ - объемный модуль, определяемый равенством

$$
K=\left.\frac{\partial^{2} E_{n}}{\partial \theta^{2}}\right|_{c=\text { const }} .
$$

В работе [7] был вычислен объемный модуль $K$ для изотропного континуума, содержащего поры

$$
K=K_{0}\left[1-\frac{3(1-\mu)}{2(1-2 \mu)} c\right],
$$

где $K_{0}$ - объемный модуль континуума в отсутствие пор, $\mu-$ коэффициент Пуассона, $c-$ концентрация пор.

Как уже упоминалось, при быстром нагреве возникают кластеры вакансий. Это обстоятельство позволяет отождествить концентрацию пор с концентрацией вакансий, после чего подстановка равенства (37) в формулу (35) приводит к уравнению состояния

$$
P=-K_{0} \theta\left[1-B\left(\theta-\theta_{c}\right) \eta\left(\theta_{c}\right)\right],
$$

где $\eta\left(\theta_{c}\right)$ - функция Хевисайда, определяемая формулой

$$
\eta\left(\theta_{c}\right)=\left\{\begin{array}{ll}
0, & \theta<\theta_{c} \\
1, & \theta>\theta_{c}
\end{array},\right.
$$

а посредством $B$ обозначено выражение

$$
B=\frac{3}{2} \frac{(1-\mu)}{(1-2 \mu)} \frac{1}{1+\theta_{c}} .
$$

Как видно из формулы (38), при $\theta=\theta_{c}$ зависимость $P(\theta)$ испытывает излом.

В точке излома $\theta_{c}$ вторая производная (36) характеристической термодинамической функции обладает особенностью. Следовательно при деформации $\theta_{c}$ в кристалле происходит фазовый переход второго рода. Исходной фазой является идеальный кристалл, вновь образовавшейся - кристалл, содержащий вакансии.

Из формулы (38) также следует, что при $\theta=\theta_{F}=\frac{1}{2 B}$ выполняется равенство $\frac{d P}{d \theta}=0$. Это равенство означает, что при $\theta>\theta_{F}$ решетка теряет устойчивость. Что происходит с кристаллом после превышения деформации $\theta_{F}$ ?

Согласно справочным данным для твердых тел с точностью до сотых можно принять, что $\mu \approx 0.4$. Тогда получаем оценку $B \approx 4.5$. При этом для $\theta_{F}$ имеем оценку $\theta_{F} \approx 0.1$. Для такой деформации существенно снижается активационный барьер $\Delta u$, вследствие чего частицы снова могут совершать поступательное движение, что приводит к разрушению неустойчивой кристаллической решетки. Способствует последнему и наличие вакансионных кластеров в решетке. Теперь кластеры получают возможность менять свою форму, соединяться друг с другом, в результате чего система оказывается пронизанной трещинами. В работе [1] жидкость представляется как совокупность разделенных трещинами плотных областей, не обладающих решеткой. Именно такой оказывается структура исследуемой в данной статье системы после того, как решетка теряет устойчивость. На этом основании можно утверждать, что при $\theta>\theta_{F}$ исследуемая система становится жидкостью.

Дополнительным аргументом в пользу этого утверждения является значение $\theta_{F}$, приблизительно равное относительному уменьшению плотности при плавлении. Из неустойчивости состояния кристалла, соответствующего $\theta=\theta_{F}$, следует, что даже крайне малой разности $\theta-\theta_{F}$ достаточно, чтобы система приобрела структуру жидкости. Это означает, что плавление происходит практически при постоянном объеме $V=V_{F}$ и что в качестве температуры плавления при быстром нагреве выступает температура $T_{F}$, определяемая из соотношения

$$
\theta\left(T_{F}\right)=\theta_{F}
$$

При этом, поскольку превращение „твердая фазажидкость“ представляет собой последовательность абсолютно неустойчивых состояний, оно носит взрывоподобный характер, протекая практически мгновенно.

\section{4. Заключение}

Проведенное в данной статье исследование, по мнению авторов позволяет утверждать, что традиционный механизм, который принято использовать для описания плавления и который связан с образованием критических зародышей и их последующим ростом, при быстром нагреве теряет свою силу. Причина заключается в том, что при быстром изменении температуры не возбуждается поступательное движение частиц, формирующее зародыши и обеспечивающее их рост.

В этих условиях вступает в действие другой механизм, согласно которому с кристаллом происходят следующие изменения. В интервале от начальной температуры $T_{B}$ до температуры $T_{c}$ кристалл испытывает упругую деформацию.

Температура $T_{c}$ - температура фазового перехода второго рода. В температурном интервале от $T_{c}$ до $T_{F}$ образуется новая фаза - кристалл, содержащий вакансионные кластеры. При $T>T_{F}$ кристаллическая решетка теряет механическую устойчивость.

Разупорядочение структуры, которое является следствием потери устойчивости, уменышая активационные 
барьеры для элементарных перемещений частиц, возобновляет поступательное движение. Благодаря последнему практически при постоянных температуре $T_{F}$ и объеме происходит разрушение неустойчивой решетки и формирование жидкостной структуры. Этот процесс представляет собой последовательность абсолютно неустойчивых состояний и поэтому носит взрывоподобный характер, протекая практически мгновенно. Температура $T_{F}$, при которой кристалл теряет механическую устойчивость выступает в качестве температуры плавления кристалла при быстром нагреве.

\section{Финансирование работы}

Работа выполнена в рамках пограммы исследований по теме № 17БФ051-01, запланированных в НИЛ „Физика жидкостей, полимеров и фазовых переходов в них“ физического факультета Киевского национального университета им. Тараса Шевченко.

\section{Конфликт интересов}

Авторы заявляют, что у них нет конфликта интересов.

\section{Список литературы}

[1] Я.И. Френкель. Кинетическая теория жидкостей. Наука, Л. (1975). $592 \mathrm{c}$.

[2] Amit Samanta' Mark E. Tuckerman, Tang-Qing Yu, Weinan E. Science 346, 6210, 729 (2014).

[3] J.M. Dealy, K.F. Wissbrun. Melt Rheology and Its Role in Plastics Processing. Theory and Applications. Kluwer Academic Publishers, Amsterdam (1999). 600 p.

[4] Л.Д. Ландау, Е.М. Лифшиц Статистическая физика. Наука, М. (1976). $584 \mathrm{c}$.

[5] Л.А. Булавин, О.Ю. Актан, Ю.Ф. Забашта. ФТТ 50, 12, 2174 (2008).

[6] Л.А. Булавин, О.Ю. Актан, Ю.Ф. Забашта. ФТТ 52, 4, 662 (2010).

[7] М.А. Криовоглаз, А.С. Черевко. Об упругом модуле твердой фазы. ФММ 8, 2, 161 (1959).

Редактор Т.Н. Василевская 